\title{
Clinico-microbiological profile of Burkholderia cepacia keratitis: a case series
}

Ming-Chih Ho ${ }^{1}$, Eugene Yu-Chuan Kang ${ }^{1}$, Lung-Kun Yeh ${ }^{1,2}$, David H. K. Ma ${ }^{1,2}$, Hsin-Chiung Lin ${ }^{1,2}$, Hsin-Yuan Tan ${ }^{1,2}$, Hung-Chi Chen ${ }^{1,2}$ and Ching-Hsi Hsiao ${ }^{1,2^{*}}$

\begin{abstract}
Background: Burkholderia cepacia, an opportunistic pathogen mainly affecting patients with cystic fibrosis or immunocompromised, has rarely been documented as a cause of corneal infection. The clinical and microbiological profiles of $B$. cepacia keratitis are reported herein.

Methods: We retrospectively reviewed the medical record of 17 patients with culture-proven B. cepacia keratitis, treated between 2000 and 2019 at Chang Gung Memorial Hospital, Taiwan. Our data included predisposing factors, clinical presentations, treatments, and visual outcomes of B. cepacia keratitis as well as the drug susceptibility of the causative agent.
\end{abstract}

Results: The most common predisposing factor for B. cepacia keratitis was preexisting ocular disease (seven, 41.2\%), particularly herpetic keratitis (five). Polymicrobial infection was detected in seven (41.2\%) eyes. All B. cepacia isolates were susceptible to ceftazidime. Main medical treatments included levofloxacin or ceftazidime. Surgical treatment was required in five (29.4\%) patients. Only four (23.5\%) patients exhibited final visual acuity better than 20/200.

Conclusions: B. cepacia keratitis primarily affects patients with preexisting ocular disease, particularly herpetic keratitis, and responds well to ceftazidime or fluoroquinolones. However, the visual outcomes are generally poor.

Keywords: Burkholderia cepacia, keratitis, corneal ulcer, drug susceptibility

\section{Background}

Burkholderia cepacia complex, formerly known as Pseudomonas cepacia, is a group of aerobic Gram-negative bacilli comprising more than 20 species $[1,2]$. It can exist in various environments, such as soil or water, and can infect both humans and plants. In humans, B. cepacia is principally an opportunistic pathogen that causes various diseases, such as lung infections, in patients with cystic fibrosis or chronic granulomatous disease. Ocular manifestations caused by B. cepacia include endophthalmitis and keratitis, both of which are vision-threatening [3-9]. Several case series have reported on B. cepacia

*Correspondence: hsiao.chinghsi@gmail.com

1 Department of Ophthalmology, Chang Gung Memorial Hospital, No.

199, Tung-Hwa North Road, Linkou, Taipei, Taiwan

Full list of author information is available at the end of the article endophthalmitis, which occurs after ocular surgery or ocular trauma. Compared with endophthalmitis, B. cepacia keratitis has rarely been reported, with only eight sporadic cases being documented thus far $[4,5,7,9$, 10]. Here, we report on 17 cases of B. cepacia keratitis. By reviewing patient demographics, risk factors, clinical presentations, treatment, and visual outcomes, we identified the characteristics of the disease, thus contributing additional knowledge on B. cepacia keratitis.

\section{Materials and methods}

This single-center retrospective study included data of 17 patients diagnosed as having B. cepacia keratitis at Chang Gung Memorial Hospital, Taiwan between December 2003 and August 2019. Corneal scrapings, obtained under topical anesthesia, were inoculated on blood and chocolate agar, thioglycolate broth, and

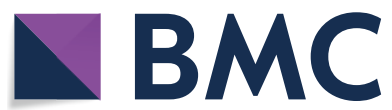

(c) The Author(s) 2021. This article is licensed under a Creative Commons Attribution 4.0 International License, which permits use, sharing, adaptation, distribution and reproduction in any medium or format, as long as you give appropriate credit to the original author(s) and the source, provide a link to the Creative Commons licence, and indicate if changes were made. The images or other third party material in this article are included in the article's Creative Commons licence, unless indicated otherwise in a credit line to the material. If material is not included in the article's Creative Commons licence and your intended use is not permitted by statutory regulation or exceeds the permitted use, you will need to obtain permission directly from the copyright holder. To view a copy of this licence, visit http://creativeco mmons.org/licenses/by/4.0/. The Creative Commons Public Domain Dedication waiver (http://creativecommons.org/publicdomain/ zero/1.0/) applies to the data made available in this article, unless otherwise stated in a credit line to the data. 
Lowenstein-Jenson agar as well as subjected to Gram staining. The various media were routinely incubated for one week or longer, depending on the medium, before the final culture result was obtained. Isolates were identified, by using conventional biochemical tests; matrix-assisted laser desorption/ionization time-of-flight (MALDI-TOF) mass spectrometry was applied starting in 2013. Antimicrobial susceptibility was evaluated using the standard disk diffusion method and interpreted according to the guidelines established by the Clinical and Laboratory Standards Institute (CLSI). For B. cepacia isolates, ceftazidime, meropenem, and sulfamethoxazole-trimethoprim were tested. Each patient's demographic data, risk factors, clinical presentations, treatments, and visual outcomes were reviewed. We provided a case report (patient 13) as a representative of B. cepacia keratitis in our study. We defined the location of an ulcer as central if it was located within $2 \mathrm{~mm}$ of fixation, periphery if it involved a zone within $2 \mathrm{~mm}$ from the limbus, and paracentral if it was in between. The ulcer size was defined as small $(<2 \mathrm{~mm})$, medium $(2-6 \mathrm{~mm})$, or large $(>6 \mathrm{~mm})$ on the basis of the longest diameter. Predisposing factors were classified into ocular trauma, contact lens wear, preexisting ocular disease, recent ocular surgery, and systemic disease. Prior steroid use was also recorded. Visual acuity was measured using Snellen charts.

\section{Results}

Table 1 lists the demographic and clinical data of the patients. The mean patient age was $62.4 \pm 17.2$ (range 24-88) years. A total of 17 eyes were involved, with nine right eyes and eight left eyes in eight male and nine female patients. Mean follow-up duration was 2.76 years (range 7 days to nine years).

Of the 17 corneal ulcers, 10 (58.8\%) were located in the central cornea. In terms of size, eight (47.1\%), four (23.5\%), and five (29.4\%) corneal ulcers were defined as large, medium, and small, respectively. Hypopyon was present in eight $(47.1 \%)$ patients. Corneal perforation was observed in four (23.5\%) patients-in two at presentation and in two during treatment.

Predisposing factors of keratitis were identified in 14 patients, with four patients demonstrating multifactorial causes of keratitis. Preexisting ocular diseases (seven eyes, $41.2 \%$ ), particularly herpetic keratitis (five eyes), was the most common predisposing factor. Other risk factors, including trauma (three eyes), systemic disease (three eyes), contact lens wear (two eyes), and recent ocular surgery (two eyes), were relatively evenly distributed. Prior corticosteroid use was noted in eight (47.1\%) patients.

Of the $17 \mathrm{~B}$. cepacia culture-positive scrapings, seven cases $(41.2 \%)$ were polymicrobial (Table 1$)$. All 17 B. cepacia isolates were susceptible to ceftazidime; all except for one $(16 / 17,94.1 \%)$ were susceptible to meropenem and sulfamethoxazole-trimethoprim.

All patients were treated with empiric topical antibiotics initially, and adjustments were made according to clinical response or culture results. Levofloxacin, ceftazidime, and amikacin, the main antibiotics for treating B. cepacia keratitis, were prescribed to nine (52.9\%), six (35.3\%), and two (11.7\%) patients, respectively. In patients with polymicrobial keratitis, other antimicrobials were added. A total of 12 patients (70.6\%) responded well to antimicrobials, whereas five patients (29.4\%) required surgical interventions including amniotic graft transplantation, patch graft, tarsorrhaphy, and evisceration. Multiple surgeries were required in three patients.

Visual acuity (VA) worse than 20/200 was noted in 14 patients $(82.4 \%)$ at presentation; moreover, four patients (23.5\%) had no light perception. After treatment, six eyes exhibited improved vision but only four patients (23.5\%) had a final VA better than 20/200.

\section{Case report (Patient 13)}

An 84-year-old female patient with herpes simplex virus disciform keratitis was undergoing treatment with topical prednisolone acetate (1\%) and oral acyclovir and exhibited sudden onset of blurred vision in her right eye one month after discontinuing the antiviral medication. On examination, VA in the right eye was hand motions. Slitlamp examination revealed corneal epithelial defect with infiltrate, thinning with a descematocele, and localized edema; strong anterior chamber reaction with hypopyon was also present (Fig. 1). Corneal scrapings were sent for cultures. She was administered on topical vancomycin $(25 \mathrm{mg} / \mathrm{mL})$ and ceftazidime $(25 \mathrm{mg} / \mathrm{mL})$ hourly and oral famciclovir three times a day. The corneal culture grew $B$. cepacia complex, susceptible to ceftazidime, meropenem, and sufamethoxazole-trimethoprim. She was maintained on topical ceftazidime, and when the infection was controlled, a topical corticosteroid was added. The ulceration resolved within 1 week. At 9-month follow-up, she had a corneal scar with VA of 20/400 in the right eye.

\section{Discussion}

B. cepacia is a rare causative agent of keratitis; only eight cases of $B$. cepacia keratitis have been reported in previous studies (Table 2). B. cepacia accounted for $0.51 \%$ $(5 / 875)$ of microbial keratitis cases in our previous tenyear (2003-2012) study [11], but we identified 12 more cases in recent years. To our best knowledge, this study is by far the largest case series related to B. cepacia keratitis. In conjunction with previously reported cases, we provided a more detailed overview of the clinical characteristics of B. cepacia keratitis. 


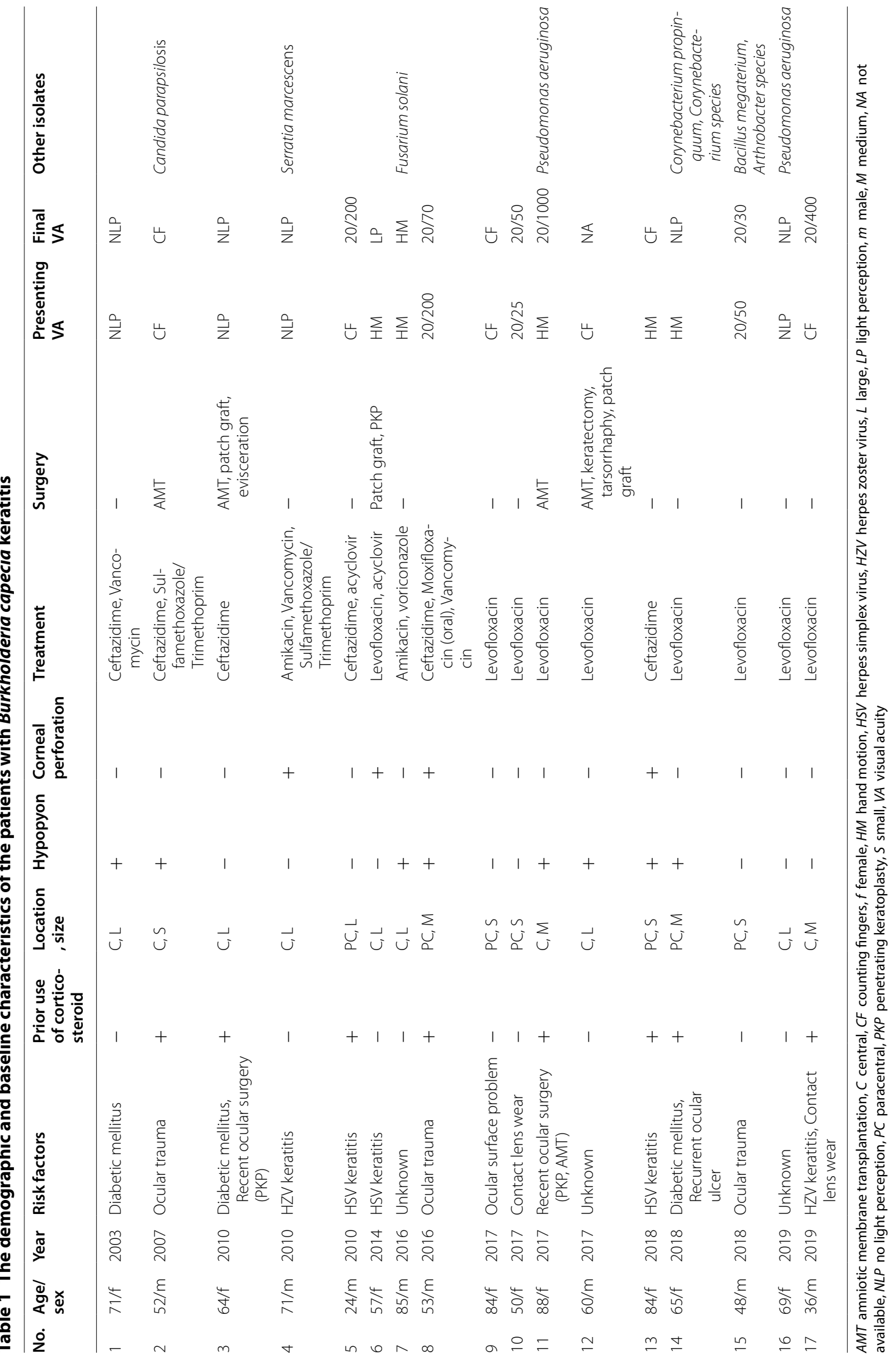




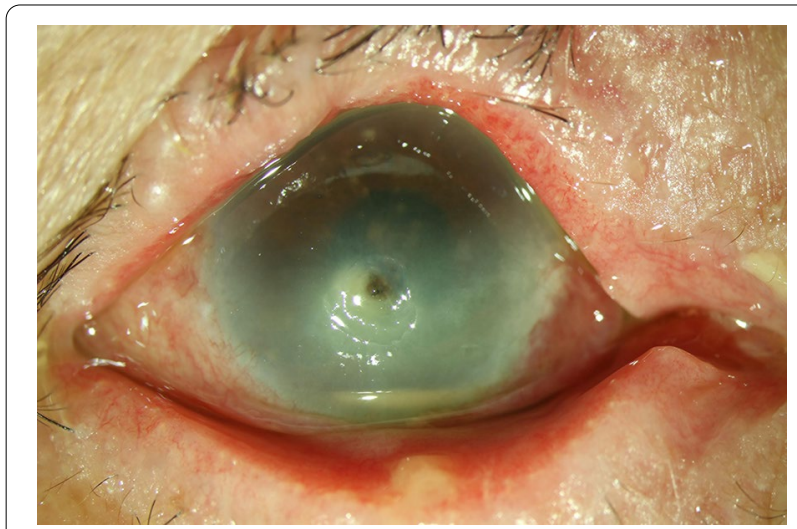

Fig. 1 The slit-lamp photograph revealed central corneal epithelial defect with infiltrate, thinning with a descemetocele, and hypopyon

In our study, the most common predisposing factor of B. cepacia keratitis was preexisting ocular disease, particularly herpetic keratitis. Matoba et al. also presented a patient with herpetic stroma keratitis, under oral acyclovir and topical prednisolone acetate treatment, who developed polymicrobial keratitis including B. ambifaria (belonging to the $B$. cepacia complex), Enterococcus spp., and Staphylococcus aureus [7]. Infection with herpes virus might cause sub-basal nerve damage of the cornea $[12,13]$. The impaired corneal sensory innervation leads to a reduction of protective reflexes and trophic neuromodulators, which affect the wound-healing function of the cornea [14], making its surface an easy target for opportunistic bacteria such as B. cepacia. In addition, if the local immune response has been suppressed by topical steroids, a herpetic corneal ulcer can predispose microbial adherence, furthering the infection. Recent ocular surgery with simultaneous topical steroid use was noted in three of the previously reported eight patients with B. cepacia keratitis and two patients in our study (Tables 1 and 2), suggesting that local immunosuppression may play a role in such an opportunistic infection.

In our study, approximately $40 \%$ of B. cepacia culture-positive corneal scrapings were polymicrobial, as were two $(25 \%)$ of the previously reported eight cases (Table 2). These mixed infections might be due to direct inoculation because of a corneal injury, contamination through the process of corneal scraping, or opportunistic transmission in these immunocompromised patients [15]. Tuft et al. proposed a synergy effect of interactions between organisms in polymicrobial infection [16] and speculated that the primary organism may create a niche, either by providing a sequestered environment or by supplying specific metabolic requirements for a second organism, that predisposes the host to further infection or turns a normally nonpathogenic organism into a

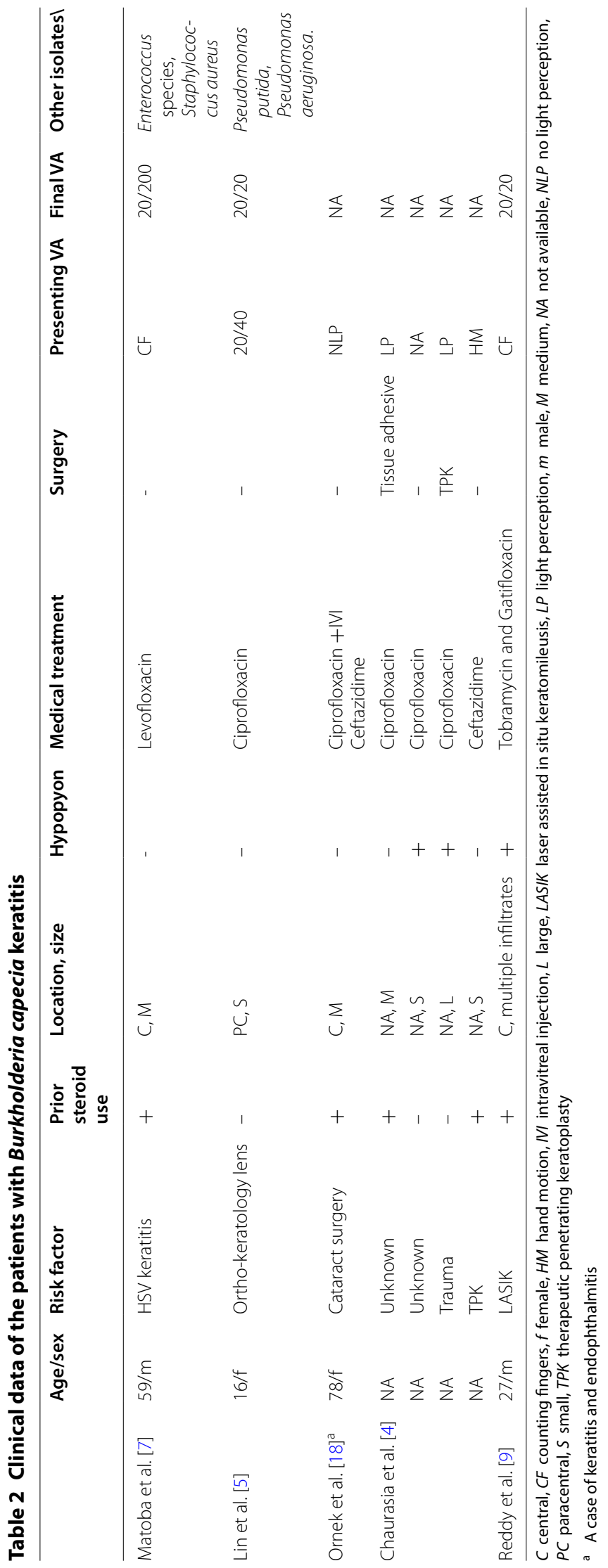


pathogen. The mixed infections might modulate the clinical course of the disease, causing unexpected treatment effects.

B. cepacia demonstrates multidrug resistance, including resistance to carboxypenicillins, polymyxins, and aminoglycosides. Nevertheless, sulfamethoxazole-trimethoprim, ceftazidime, and meropenem have been revealed to be the most effective agents on the basis of in vitro susceptibility data, which agrees with our drug susceptibility test results [17]. We did not test for susceptibility to fluoroquinolones, the most popular empiric antibiotic in the field of ophthalmology. Chaurasia et al. performed an antibiotic susceptibility test for four B. cepacia isolated from keratitis and reported $100 \%$ susceptibility to ceftazidime and $50 \%$ susceptibility to ciprofloxacin/norfloxacin [4]. In the case report by Reddy et al. the isolate from the patient with B. cepacia keratitis was resistant to moxifloxacin, gatifloxacin, tobramycin, and ceftazidime and susceptible only to sulfamethoxazole-trimethoprim in vitro; nevertheless, in vivo, the ulcer resolved completely after tobramycin and gatifloxacin treatment (Table 2) [9]. The other three isolates from previously reported B. cepacia keratitis cases were susceptible to ceftazidime and ciprofloxacin $[5,7,18]$. On the basis of the antibiotic susceptibility and clinical results of the patients with $B$. cepacia keratitis (Tables 1 and 2), fluoroquinolones could be initiated as empiric antibiotics. However, if fluoroquinolone use does not improve the clinical course, ceftazidime may be a suitable alternative. Even after aggressive medical treatment, about one-third of the patients in our study and two (25\%) of the previously reported eight $B$. cepacia keratitis cases required surgical interventions (Tables 1 and 2).

The visual outcome of B. cepacia keratitis was generally poor both in our and previously reported cases (Tables 1 and 2). The unfavorable visual outcomes may be related to old age, poor vision at presentation, comorbidities, and mixed infections. The rather high surgical rates and perforation rates may also contribute to the poor prognosis of the disease.

The retrospective design and small sample size are the limitations of this study. In addition, elucidating the real pathogenic role of B. cepacia was difficult because polymicrobial infections were detected in approximately $40 \%$ of our patients. Nevertheless, as the largest case series reporting $B$. cepacia keratitis, this study provides more detailed information regarding the clinical and microbiological profiles of this infection.

In conclusion, although relatively uncommon, $B$. cepacia could be a causative agent of infectious keratitis. Our findings revealed that preexisting ocular disease, particularly herpetic keratitis, was the leading predisposing factor of $B$. cepacia keratitis. B. cepacia demonstrated clinical response to the treatment of ceftazidime and fluoroquinolone, but some patients required surgical intervention. However, the visual outcome was generally poor.

\begin{abstract}
Abbreviation
VA: Visual acuity.

Authors' contributions

All authors have participated directly in planning and execution of the work. MCH, EYCK: acquisition and analysis of data, drafting and writing the article; LKY, DHKM, HCL, HYT, HCC: acquisition and analysis of data; CHH: design of the study, acquisition of data, final approval. All authors read and approved the final manuscript.

Funding

None.

Availability of data and materials

The data analyzed during this study are available on request from the corresponding author, Ching-Hsi Hsiao. The data are not publicly available due to it containing information that could compromise the privacy of research participants.
\end{abstract}

\section{Ethics approval and consent to participate}

The study adhered to the Declaration of Helsinki and was approved by the Institutional Review Board of Chang Gung Memorial Hospital (IRB number: 202000181B0), which granted a waiver of consent because patient anonymity was maintained by the data source.

\section{Consent for publication}

The consent for publication of biometric data from Patient 13 was obtained.

\section{Competing interests}

The authors declare that they have no competing interests.

\section{Author details}

1 Department of Ophthalmology, Chang Gung Memorial Hospital, No. 199, Tung-Hwa North Road, Linkou, Taipei, Taiwan. ${ }^{2}$ College of Medicine, Chang Gung University, Taoyuan, Taiwan.

Received: 1 May 2020 Accepted: 3 December 2020

Published online: 07 January 2021

\section{References}

1. Mahenthiralingam E, Urban TA, Goldberg JB. The multifarious, multireplicon Burkholderia cepacia complex. Nat Rev Microbiol. 2005;3::144-56.

2. LiPuma JJ. Update on the Burkholderia cepacia complex. Curr Opin Pul Medicine. 2005;11:528-33.

3. Sachdeva V, Joseph V, Sharma S, Das T. Burkholderia cepacia Endophthalmitis. Clinico-Microbiologic Profile Outcomes Retina. 2011;31:1801-5.

4. Chaurasia S, Muralidhar R, Das S, Sangwan VS, Reddy AK, Gopinathan U. Keratitis Caused by Burkholderia cepacia. Br J Ophthalmol. 2011;95::746-7.

5. Lin YC, Chen KH, Hsu WM. Daytime orthokeratology associated with infectious keratitis by multiple gram-negative bacilli: Burkholderia cepacia, Pseudomonas putida, and Pseudomonas aeruginosa. Eye Contact Lens. 2006;32:19-20.

6. Eser I, Altan T, Stahl J, Aydin M, Inan N, Kapran Z. Yilmaz O. Two cases of Burkholderia cepacia endophthalmitis. Br J Ophthalmol 2006; 90: 1211.

7. Matoba AY. Polymicrobial keratitis secondary to Burkholderia ambifaria, enterococcus, and staphylococcus aureus in a patient with herpetic stromal keratitis. Br J Ophthalmol. 2003;136:748-9. 
8. Pathengay A, Raju B, Sharma S, Das T. Recurrent endophthalmitis caused by Burkholderia cepacia. Eye. 2005;19:358.

9. Reddy JC, Tibbetts MD, Hammersmith KM, Nagra PK, Rapuano CJ. Successful management of Burkholderia cepacia keratitis after LASIK. J Refract Surg. 2013;29:8-9.

10. Wright TM, Afshari NA. Microbial keratitis following corneal transplantation. Am J Ophthalmol. 2006:142:1061-2.

11. Hsiao CH, Sun CC, Yeh LK, Ma DH, Chen PY, Lin HC, Tan HY, Chen HC, Chen SY, Huang YC. Shifting trends in bacterial keratitis in Taiwan: a 10-year review in a tertiary-care hospital. Cornea. 2016;35:313-7.

12. Danileviciene V, Zemaitiene R, Gintauskiene VM, Nedzelskiene I, Zaliuniene D. Corneal sub-basal nerve changes in patients with herpetic keratitis during acute phase and after 6 months. Medicina. 2019;55:214.

13. Versura P, Giannaccare G, Pellegrini M, Sebastiani S, Campos EC. Neurotrophic keratitis: current challenges and future prospects. Eye Brain. 2018:10:37-45.
14. Sousa SA, Ramos CG, Leitao JH. Burkholderia cepacia complex: emerging multihost pathogens equipped with a wide range of virulence factors and determinants. Int J Microbiol. 2011;607575:9.

15. Ahn M, Yoon KC, Ryu SK, Cho NC, You IC. Clinical aspects and prognosis of mixed microbial (bacterial and fungal) keratitis. Cornea. 2011;30:409-13.

16. Tuft S. Polymicrobial infection and the eye. Br J Ophthalmol. 2006;90:257-8.

17. Sfeir MM. Burkholderia cepacia complex infections: more complex than the bacterium name suggest. J Infect. 2018:77:166-70.

18. Ornek K, Ozdemir M, Ergin A. Burkholderia cepacia keratitis with endophthalmitis. J Med Microbiol. 2009;58:1517-8.

\section{Publisher's Note}

Springer Nature remains neutral with regard to jurisdictional claims in published maps and institutional affiliations.
Ready to submit your research? Choose BMC and benefit from:

- fast, convenient online submission

- thorough peer review by experienced researchers in your field

- rapid publication on acceptance

- support for research data, including large and complex data types

- gold Open Access which fosters wider collaboration and increased citations

- maximum visibility for your research: over $100 \mathrm{M}$ website views per year

At BMC, research is always in progress.

Learn more biomedcentral.com/submissions 